\title{
GER-TYPE AND HYERS-ULAM STABILITIES FOR THE FIRST-ORDER LINEAR DIFFERENTIAL OPERATORS OF ENTIRE FUNCTIONS
}

\author{
TAKESHI MIURA, GO HIRASAWA, and SIN-EI TAKAHASI
}

Received 22 April 2003

Dedicated to Professor Seiji Watanabe on his 60th birthday (Kanreki).

\begin{abstract}
Let $h$ be an entire function and $T_{h}$ a differential operator defined by $T_{h} f=f^{\prime}+h f$. We show that $T_{h}$ has the Hyers-Ulam stability if and only if $h$ is a nonzero constant. We also consider Ger-type stability problem for $\left|1-f^{\prime} / h f\right| \leq \varepsilon$.
\end{abstract}

2000 Mathematics Subject Classification: 34K20, 26D10.

1. Introduction. The first result, which we now call the Hyers-Ulam stability (HUS), is due to Hyers [4] who gave an answer to a question posed by Ulam (cf. [11, Chapter VI] and [12]) in 1940 concerning the stability of homomorphisms: for what metric groups $G$ is it true that an $\varepsilon$-automorphism of $G$ is necessarily near to a strict automorphism?

An answer to the above problem has been given as follows. Suppose $E_{1}$ and $E_{2}$ are two real Banach spaces and $f: E_{1} \rightarrow E_{2}$ is a mapping such that $f(t x)$ is continuous in $t \in \mathbb{R}$, the set of all real numbers, for each fixed $x \in E_{1}$. If there exist $\theta \geq 0$ and $p \in \mathbb{R} \backslash\{1\}$ such that

$$
\|f(x+y)-f(x)-f(y)\| \leq \theta\left(\|x\|^{p}+\|y\|^{p}\right)
$$

for all $x, y \in E_{1}$, then there is a unique linear mapping $T: E_{1} \rightarrow E_{2}$ such that $\| f(x)-$ $T(x)\|\leq 2 \theta\| x \|^{p} /\left|2-2^{p}\right|$ for every $x \in E_{1}$. Hyers [4] obtained the result for $p=0$. Then Rassias [7] generalized the above result of Hyers to the case where $0 \leq p<1$, while the proof given in [7] also works for $p<0$. Gajda [2] solved the problem for $1<p$ and also gave an example that a similar result does not hold for $p=1$ (cf. [8]).

In connection with the stability of exponential functions, Alsina and Ger [1] remarked that the differential equation $y^{\prime}=y$ has the HUS. More explicitly, suppose $I$ is an open interval, $\varepsilon>0$, and $f: I \rightarrow \mathbb{R}$ is a differentiable function such that $\left|f^{\prime}(t)-f(t)\right| \leq \varepsilon$ for all $t \in I$. Then, there is a differentiable function $g: I \rightarrow \mathbb{R}$ such that $g^{\prime}=g$ and $|f(t)-g(t)| \leq 3 \varepsilon$ for all $t \in I$. The third and first authors of this paper along with Miyajima [10] considered the Banach-space-valued differential equation $y^{\prime}=\lambda y$, where $\lambda$ is a complex constant. Then they proved the HUS of $y^{\prime}=\lambda y$ under the condition that $\operatorname{Re} \lambda \neq 0$. Though, they treated the result as the stability of the operator $D-I_{d}$, where $D$ denotes the ordinary differential operator and $I_{d}$ the identity. Some stability results of other differential equations (or operators) are also known (cf. [5, 6, 9]). 
Taking the group structure of $\mathbb{C} \backslash\{0\}$ into account, Ger and Šemrl [3] considered the inequality

$$
\left|\frac{f(x+y)}{f(x) f(y)}-1\right| \leq \theta \quad(x, y \in S)
$$

for a mapping $f: S \rightarrow \mathbb{C} \backslash\{0\}$, where $(S,+)$ is a semigroup and $\mathbb{C}$ is the set of all complex numbers. If $0 \leq \theta<1$ and if $(S,+)$ is a cancellative abelian semigroup, then they proved that there is a unique function $g: S \rightarrow \mathbb{C} \backslash\{0\}$ such that $g(x+y)=g(x) g(y)$ for all $x, y \in S$ and that

$$
\max \left\{\left|\frac{f(x)}{g(x)}-1\right|,\left|\frac{g(x)}{f(x)}-1\right|\right\} \leq \sqrt{1+\frac{1}{(1-\theta)^{2}}-2 \sqrt{\frac{1+\theta}{1-\theta}}}
$$

for all $x \in S$. The stability phenomena of this kind is called Ger-type stability.

Throughout this paper, $H(\mathbb{C})$ stands for the set of all entire functions. Let $h \in H(\mathbb{C})$ and $T_{h}: H(\mathbb{C}) \rightarrow H(\mathbb{C})$ be a linear differential operator defined by

$$
T_{h} f(z)=f^{\prime}(z)+h(z) f(z) \quad(f \in H(\mathbb{C}), z \in \mathbb{C}) .
$$

DEFINITION 1.1. The operator $T_{h}$ is said to have the HUS if and only if there exists a constant $K \geq 0$ with the following property: to each $\varepsilon \geq 0$ and $f, g \in H(\mathbb{C})$ satisfying $\sup _{z \in \mathbb{C}}\left|T_{h} f(z)-g(z)\right| \leq \varepsilon$, there exists an $f_{0} \in H(\mathbb{C})$ such that $T_{h} f_{0}=g$ and $\sup _{z \in \mathbb{C}}\left|f(z)-f_{0}(z)\right| \leq K \varepsilon$. Such $K$ is called an HUS constant for $T_{h}$. If, in addition, the minimum of all such $K$ 's exists, then it is called the HUS constant for $T_{h}$.

In this paper, we first consider the HUS of the differential operator $T_{h}$. Then we show that $T_{h}$ has the HUS if and only if $h \in H(\mathbb{C})$ is a nonzero constant function. Moreover, we give the HUS constant for $T_{h}$. Finally, we consider the Ger-type stability problem of the differential equation $y^{\prime}=\lambda y$. To be more explicit, suppose $\varepsilon \geq 0$ and $f \in H(\mathbb{C})$ satisfies

$$
\sup _{z \in \mathbb{C}}\left|\frac{f^{\prime}(z)}{\lambda f(z)}-1\right| \leq \varepsilon .
$$

Does there exist $K \geq 0$ such that

$$
\sup _{z \in \mathbb{C}}\left|\frac{f(z)}{c e^{\lambda t}}-1\right| \leq K \varepsilon \quad \text { or } \quad \sup _{z \in \mathbb{C}}\left|\frac{c e^{\lambda t}}{f(z)}-1\right| \leq K \varepsilon
$$

holds for some $c \in \mathbb{C} \backslash\{0\}$ ? To this problem, we give a negative answer: the Ger-type stability does not hold in general. Moreover, we show that the solution $f \in H(\mathbb{C})$ to the differential equation $y^{\prime}=\lambda y$ is only the function which satisfies both (1.5) and (1.6).

2. The HUS for $T_{h}$. For simplicity, we write $\int_{0}^{z} f(\zeta) d \zeta$ for $\int_{0}^{1} f(z t) z d t$, where $z \in \mathbb{C}$ and $f \in H(\mathbb{C})$. We associate to each $h \in H(\mathbb{C})$ a function $\tilde{h}$ defined by

$$
\tilde{h}(z)=\exp \int_{0}^{z} h(\zeta) d \zeta \quad(z \in \mathbb{C})
$$


Let $h \in H(\mathbb{C})$. Throughout this section, $T_{h}: H(\mathbb{C}) \rightarrow H(\mathbb{C})$ denotes a linear differential operator defined by (1.4). Suppose $f, g \in H(\mathbb{C})$. Then note that $T_{h} f=g$ if and only if $f$ is of the form

$$
f(z)=\frac{1}{\tilde{h}(z)}\left\{f(0)+\int_{0}^{z} g(\zeta) \tilde{h}(\zeta) d \zeta\right\} \quad(z \in \mathbb{C}) .
$$

LEMMA 2.1. Suppose $h \in H(\mathbb{C})$ is not a constant function, $f \in H(\mathbb{C})$, and

$$
0<\sup _{z \in \mathbb{C}}\left|T_{h} f(z)\right|<\infty .
$$

Then

$$
\sup _{z \in \mathbb{C}}\left|f(z)-\frac{c}{\tilde{h}(z)}\right|=\infty
$$

for every $c \in \mathbb{C}$.

Proof. By hypothesis, $T_{h} f$ is a bounded entire function, and so $T_{h} f$ must be constant, say $c_{0} \in \mathbb{C} \backslash\{0\}$ by Liouville's theorem. Hence, by (2.2), $f$ is of the form

$$
f(z)=\frac{1}{\tilde{h}(z)}\left\{f(0)+c_{0} \int_{0}^{z} \tilde{h}(\zeta) d \zeta\right\} \quad(z \in \mathbb{C}) .
$$

Suppose $\sup _{z \in \mathbb{C}}\left|f(z)-c_{1} / \tilde{h}(z)\right|<\infty$ for some $c_{1} \in \mathbb{C}$. Another application of Liouville's theorem yields the existence of a constant $c_{2} \in \mathbb{C}$ such that $c_{2}=f-c_{1} / \tilde{h}$, and therefore (2.5) gives

$$
c_{2} \tilde{h}(z)=f(0)-c_{1}+c_{0} \int_{0}^{z} \tilde{h}(\zeta) d \zeta \quad(z \in \mathbb{C}) .
$$

By differentiating both sides of (2.6) with respect to $z$, we obtain

$$
c_{2} h \tilde{h}=c_{0} \tilde{h}
$$

and hence

$$
c_{2} h=c_{0}
$$

Since $h$ is not constant, this implies that $c_{2}=0$. Thus, $f=c_{1} / \tilde{h}$, and hence $T_{h} f=0$ (see (2.2)), which contradicts $0<\sup _{z \in \mathbb{C}}\left|T_{h} f(z)\right|$.

THEOREM 2.2. If $h \in H(\mathbb{C})$, then each of the following statements implies the other:

(a) $h$ is a nonzero constant function,

(b) $T_{h}$ has the HUS.

Proof. (a) $\Rightarrow(\mathrm{b})$. Suppose $h$ is a nonzero constant function, say $\lambda \in \mathbb{C} \backslash\{0\}$. Then, $\tilde{h}(z)=e^{\lambda z}$ for $z \in \mathbb{C}$. Suppose $\varepsilon \geq 0$ and $f, g \in H(\mathbb{C})$ satisfy $\sup _{z \in \mathbb{C}}\left|T_{h} f(z)-g(z)\right| \leq \varepsilon$. Then there exists a $c_{0} \in \mathbb{C}$ such that $T_{h} f-g=c_{0}$ by Liouville's theorem. Put

$$
u(z)=e^{-\lambda z}\left\{\int_{0}^{z} g(\zeta) e^{\lambda \zeta} d \zeta\right\} \quad(z \in \mathbb{C}) .
$$


Then $T_{h} u=g$, and so $T_{h}(f-u)=c_{0},\left|c_{0}\right| \leq \varepsilon$. Hence, by (2.2), $f$ is of the form

$$
\begin{aligned}
f(z) & =u(z)+\frac{1}{\tilde{h}(z)}\left\{f(0)-u(0)+c_{0} \int_{0}^{z} \tilde{h}(\zeta) d \zeta\right\} \\
& =\frac{c_{0}}{\lambda}+u(z)+\left(f(0)-u(0)-\frac{c_{0}}{\lambda}\right) e^{-\lambda z}
\end{aligned}
$$

for all $z \in \mathbb{C}$. Put

$$
f_{0}(z)=u(z)+\left(f(0)-u(0)-\frac{c_{0}}{\lambda}\right) e^{-\lambda z} \quad(z \in \mathbb{C}),
$$

then $T_{h} f_{0}=g$ and

$$
\left|f(z)-f_{0}(z)\right|=\left|\frac{c_{0}}{\lambda}\right| \leq \frac{\varepsilon}{|\lambda|}
$$

for every $z \in \mathbb{C}$ so that $T_{h}$ has the HUS with an HUS constant $1 /|\lambda|$.

(b) $\Rightarrow$ (a). Put

$$
f_{1}(z)=\frac{1}{\tilde{h}(z)} \int_{0}^{z} \tilde{h}(\zeta) d \zeta \quad(z \in \mathbb{C})
$$

Then we obtain $T_{h} f_{1}=1$. Let $K<\infty$ be an HUS constant for $T_{h}$. Since $T_{h}$ has the HUS, there is an $f_{2} \in H(\mathbb{C})$, such that $T_{h} f_{2}=0$ and

$$
\sup _{z \in \mathbb{C}}\left|f_{1}(z)-f_{2}(z)\right| \leq K
$$

Note that $f_{2}$ is of the form $f_{2}(z)=f_{2}(0) / \tilde{h}(z)$ for all $z \in \mathbb{C}$, since $T_{h} f_{2}=0$. Lemma 2.1, applied to $f_{1}$, yields that $h$ is a constant function. If $h$ were 0 , then (2.13) would be written in the form $f_{1}(z)=z$ for $z \in \mathbb{C}$, and hence from (2.14), $\sup _{z \in \mathbb{C}}\left|z-f_{2}(0)\right| \leq K<\infty$, which is a contradiction. Thus, we conclude that $h$ is a nonzero constant function.

TheOREM 2.3. Suppose $\lambda \in \mathbb{C} \backslash\{0\}, f, g \in H(\mathbb{C})$, and $\sup _{z \in \mathbb{C}}\left|T_{\lambda} f(z)-g(z)\right|<\infty$. Then there exists a unique $f_{0} \in H(\mathbb{C})$ such that $T_{\lambda} f_{0}=g$ and

$$
\sup _{z \in \mathbb{C}}\left|f(z)-f_{0}(z)\right|<\infty .
$$

Furthermore, $1 /|\lambda|$ is the HUS constant for $T_{\lambda}$.

Proof. The existence of such a function $f_{0} \in H(\mathbb{C})$ is proved by Theorem 2.2, and so we need to show only the uniqueness. Suppose $f_{1} \in H(\mathbb{C})$ and $f_{2} \in H(\mathbb{C})$ satisfy $T_{\lambda} f_{j}=g$ and

$$
\sup _{z \in \mathbb{C}}\left|f(z)-f_{j}(z)\right|<\infty
$$

for $j=1$, 2. Since $T_{\lambda} f_{j}=g$,

$$
f_{j}(z)=e^{-\lambda z}\left\{f_{j}(0)+\int_{0}^{z} g(\zeta) e^{\lambda \zeta} d \zeta\right\} \quad(z \in \mathbb{C})
$$


for $j=1,2$, and hence

$$
f_{1}(z)-f_{2}(z)=\left(f_{1}(0)-f_{2}(0)\right) e^{-\lambda z} \quad \forall z \in \mathbb{C}
$$

It follows from (2.16) that $f_{1}-f_{2}$ is constant by Liouville's theorem. Therefore, $f_{1}(0)=$ $f_{2}(0)$ by (2.18), which implies that $f_{1}=f_{2}$, proving the uniqueness.

We show that $1 /|\lambda|$ is the HUS constant for $T_{\lambda}$. Indeed, $1 /|\lambda|$ is an HUS constant by (2.12). Conversely, let $K$ be an arbitrary HUS constant for $T_{\lambda}$, and put

$$
f_{2}(z)=\frac{1}{\lambda}-\frac{1}{\lambda} e^{-\lambda z} \quad(z \in \mathbb{C}) .
$$

A simple calculation shows that $f_{2}^{\prime}(z)+\lambda f_{2}(z)=1$ for all $z \in \mathbb{C}$, and hence $\sup _{z \in \mathbb{C}}\left|T_{\lambda} f_{2}(z)\right|=1$. Then, there exists an $f_{3} \in H(\mathbb{C})$ such that $T_{\lambda} f_{3}=0$ and $\sup _{z \in \mathbb{C}} \mid f_{2}(z)$ $-f_{3}(z) \mid \leq K$. Since $\left|f_{2}(z)+\lambda^{-1} e^{-\lambda z}\right|=1 /|\lambda|$ for $z \in \mathbb{C}$, the uniqueness implies that $f_{3}(z)=-\lambda^{-1} e^{-\lambda z}$, which proves $1 /|\lambda| \leq K$. Thus, $1 /|\lambda|$ is the HUS constant for $T_{\lambda}$.

3. Stability for the Ger-type differential inequality. In this section, we consider the Ger-type stability problem. First, we show that the Ger-type stability does not hold in general. Indeed, the following proposition is true.

Proposition 3.1. For $\lambda \in \mathbb{C} \backslash\{0\}$ and $\varepsilon>0$, there exists an $f \in H(\mathbb{C})$ with the following properties:

$$
\begin{gathered}
\sup _{z \in \mathbb{C}}\left|\frac{f^{\prime}(z)}{\lambda f(z)}-1\right| \leq \varepsilon, \\
\sup _{z \in \mathbb{C}}\left|\frac{f(z)}{c e^{\lambda z}}-1\right|=\sup _{z \in \mathbb{C}}\left|\frac{c e^{\lambda z}}{f(z)}-1\right|=\infty \quad \forall c \in \mathbb{C} \backslash\{0\} .
\end{gathered}
$$

Proof. We associate to each $\lambda \in \mathbb{C} \backslash\{0\}$ and $\varepsilon>0$ a function $f$ defined by

$$
f(z)=e^{(\lambda+|\lambda| \varepsilon) z} \quad(z \in \mathbb{C}) .
$$

As above, we obtain

$$
f^{\prime}(z)=(\lambda+|\lambda| \varepsilon) f(z) \quad(z \in \mathbb{C})
$$

so that

$$
\left|\frac{f^{\prime}(z)}{\lambda f(z)}-1\right|=\varepsilon \quad \forall z \in \mathbb{C} .
$$

If $c \in \mathbb{C} \backslash\{0\}$, then we have

$$
\begin{aligned}
& \left|\frac{f(z)}{c e^{\lambda z}}-1\right| \geq \frac{1}{|c|}\left|e^{|\lambda| \varepsilon z}\right|-1 \rightarrow \infty \quad(\operatorname{Re} z \longrightarrow \infty), \\
& \left|\frac{c e^{\lambda z}}{f(z)}-1\right| \geq|c|\left|e^{-|\lambda| \varepsilon z}\right|-1 \rightarrow \infty \quad(\operatorname{Re} z \longrightarrow-\infty)
\end{aligned}
$$


and so

$$
\sup _{z \in \mathbb{C}}\left|\frac{f(z)}{c e^{\lambda z}}-1\right|=\sup _{z \in \mathbb{C}}\left|\frac{c e^{\lambda z}}{f(z)}-1\right|=\infty \quad \forall c \in \mathbb{C} \backslash\{0\}
$$

One might ask when the Ger-type stability is true. We give an answer to this question. If the Ger-type stability holds, then the function $f \in H(\mathbb{C})$ must be of the form $f(z)=$ $f(0) e^{\lambda z}$. That is, the only solution to the differential equation $y^{\prime}=\lambda y$ has the Ger-type stability.

THEOREM 3.2. Suppose $\lambda \in \mathbb{C} \backslash\{0\}, \varepsilon>0$, and $f \in H(\mathbb{C})$ satisfies $f(z) \neq 0$ for all $z \in \mathbb{C}$ and (1.5) holds. Suppose

$$
\sup _{z \in \mathbb{C}}\left|\frac{f(z)}{c e^{\lambda z}}-1\right| \quad \text { or } \sup _{z \in \mathbb{C}}\left|\frac{c e^{\lambda z}}{f(z)}-1\right|
$$

is finite for some $c \in \mathbb{C} \backslash\{0\}$; then $f$ is of the form $f(z)=f(0) e^{\lambda z}$ for all $z \in \mathbb{C}$.

Proof. It follows from (1.5) that $1-f^{\prime} / \lambda f$ is constant, say $c_{0} \in \mathbb{C}$, by Liouville's theorem. Thus, $f^{\prime}=\left(1-c_{0}\right) \lambda f$, and hence

$$
f(z)=f(0) e^{\left(1-c_{0}\right) \lambda z} \quad(z \in \mathbb{C}) .
$$

Suppose that there is a $c_{1} \in \mathbb{C} \backslash\{0\}$ such that

$$
\sup _{z \in \mathbb{C}}\left|\frac{f(z)}{c_{1} e^{\lambda z}}-1\right|<\infty .
$$

From (3.8), it follows that

$$
\sup _{z \in \mathbb{C}}\left|\frac{f(0)}{c_{1}} e^{-c_{0} \lambda z}-1\right|<\infty
$$

and hence $c_{0}$ must be 0 , proving $f(z)=f(0) e^{\lambda z}$ for all $z \in \mathbb{C}$.

Similarly, we can treat the case where

$$
\sup _{z \in \mathbb{C}}\left|\frac{c_{2} e^{\lambda z}}{f(z)}-1\right|<\infty
$$

for some $c_{2} \in \mathbb{C} \backslash\{0\}$, and so the proof is omitted.

Thus far, we have treated entire functions. Finally, we consider the Ger-type stability problem in the category of holomorphic functions on a bounded region.

TheOREM 3.3. Let $0 \in \Omega$ be a bounded convex region of $\mathbb{C}$ and put $M=\sup _{z \in \Omega}|z|$. Suppose $\lambda \in \mathbb{C} \backslash\{0\}, 0 \leq \varepsilon \leq 1$, and $f: \Omega \rightarrow \mathbb{C}$ is holomorphic such that $f(z) \neq 0$ for all $z \in \Omega$ and

$$
\sup _{z \in \Omega}\left|\frac{f^{\prime}(z)}{\lambda f(z)}-1\right| \leq \varepsilon .
$$


Then there are $K_{\lambda}>0$ and $c \in \mathbb{C} \backslash\{0\}$ such that

$$
\max \left\{\sup _{z \in \Omega}\left|\frac{f(z)}{c e^{\lambda z}}-1\right|, \sup _{z \in \Omega}\left|\frac{c e^{\lambda z}}{f(z)}-1\right|\right\} \leq K_{\lambda} \varepsilon .
$$

Proof. Put $g(z)=-1+f^{\prime}(z) / \lambda f(z)$ for $z \in \Omega$, and so

$$
f^{\prime}(z)=\lambda(1+g(z)) f(z) \quad(z \in \Omega) .
$$

From (3.14), it follows that

$$
f(z)=f(0) e^{\lambda z} \exp \int_{0}^{z} \lambda g(\zeta) d \zeta
$$

for every $z \in \Omega$, and hence

$$
\begin{aligned}
\left|\frac{f(z)}{f(0) e^{\lambda z}}-1\right| & =\left|\exp \int_{0}^{z} \lambda g(\zeta) d \zeta-1\right| \leq \sum_{n=1}^{\infty} \frac{1}{n !}\left|\int_{0}^{z} \lambda g(\zeta) d \zeta\right|^{n} \\
& \leq \sum_{n=1}^{\infty} \frac{|\lambda \varepsilon z|^{n}}{n !} \leq\left(e^{|\lambda| M}-1\right) \varepsilon
\end{aligned}
$$

for all $z \in \Omega$. Similarly, we can show that

$$
\sup _{z \in \Omega}\left|\frac{f(0) e^{\lambda z}}{f(z)}-1\right| \leq\left(e^{|\lambda| M}-1\right) \varepsilon,
$$

and so the proof is complete.

ACKNOWLEDGMENT. The first and third authors are partially supported by the Grant-in-Aid for Scientific Research, Japan Society for the Promotion of Science.

\section{REFERENCES}

[1] C. Alsina and R. Ger, On some inequalities and stability results related to the exponential function, J. Inequal. Appl. 2 (1998), no. 4, 373-380.

[2] Z. Gajda, On stability of additive mappings, Int. J. Math. Math. Sci. 14 (1991), no. 3, 431434.

[3] R. Ger and P. Šemrl, The stability of the exponential equation, Proc. Amer. Math. Soc. 124 (1996), no. 3, 779-787.

[4] D. H. Hyers, On the stability of the linear functional equation, Proc. Natl. Acad. Sci. USA 27 (1941), 222-224.

[5] T. Miura, S. Miyajima, and S.-E. Takahasi, A characterization of Hyers-Ulam stability of first order linear differential operators, J. Math. Anal. Appl. 286 (2003), no. 1, 136-146.

[6] _ Hyers-Ulam stability of linear differential operator with constant coefficients, Math. Nachr. 258 (2003), 90-96.

[7] T. M. Rassias, On the stability of the linear mapping in Banach spaces, Proc. Amer. Math. Soc. 72 (1978), no. 2, 297-300.

[8] T. M. Rassias and P. Šemrl, On the behavior of mappings which do not satisfy Hyers-Ulam stability, Proc. Amer. Math. Soc. 114 (1992), no. 4, 989-993.

[9] H. Takagi, T. Miura, and S.-E. Takahasi, Essential norms and stability constants of weighted composition operators on $C(X)$, Bull. Korean Math. Soc. 40 (2003), no. 4, 583-591. 
[10] S.-E. Takahasi, T. Miura, and S. Miyajima, On the Hyers-Ulam stability of the Banach spacevalued differential equation $y^{\prime}=\lambda y$, Bull. Korean Math. Soc. 39 (2002), no. 2, 309315.

[11] S. M. Ulam, A Collection of Mathematical Problems, Interscience Tracts in Pure and Applied Mathematics, no. 8, Interscience Publishers, New York, 1960.

[12] _ _ Sets, Numbers, and Universes: Selected Works, The MIT Press, Massachusetts, 1974.

Takeshi Miura: Department of Basic Technology, Applied Mathematics and Physics, Yamagata University, Yonezawa 992-8510, Japan

E-mail address: mi ura@yz.yamagata-u.ac.jp

Go Hirasawa: Department of Mathematics, Nippon Institute of Technology, Miyashiro, Saitama 345-8501, Japan

E-mail address: hi rasawa1@muh. big1obe.ne.jp

Sin-Ei Takahasi: Department of Basic Technology, Applied Mathematics and Physics, Yamagata University, Yonezawa 992-8510, Japan

E-mail address: sin-ei@emperor.yz.yamagata-u.ac.jp 


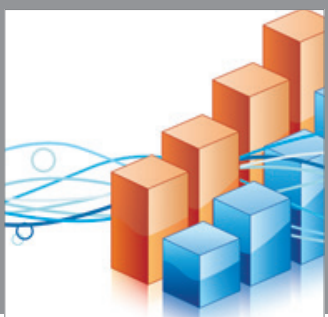

Advances in

Operations Research

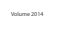

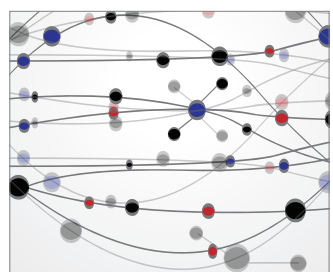

\section{The Scientific} World Journal
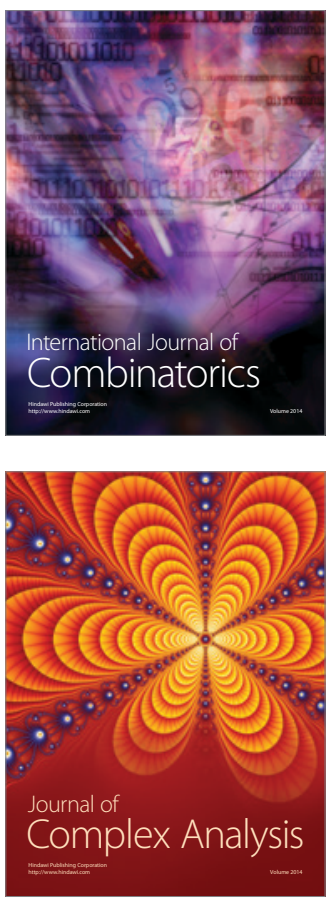

International Journal of

Mathematics and

Mathematical

Sciences
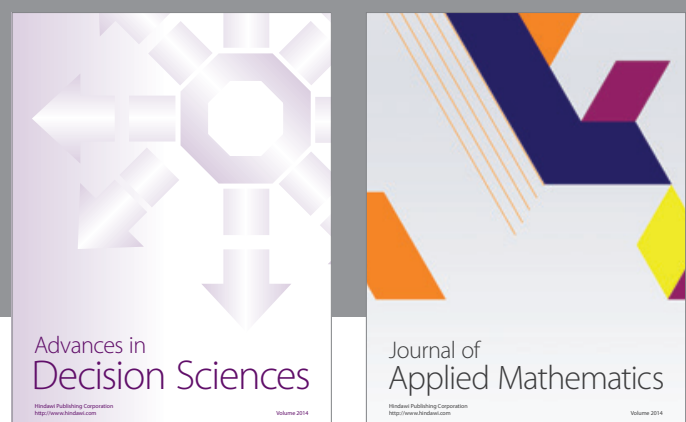

Journal of

Applied Mathematics
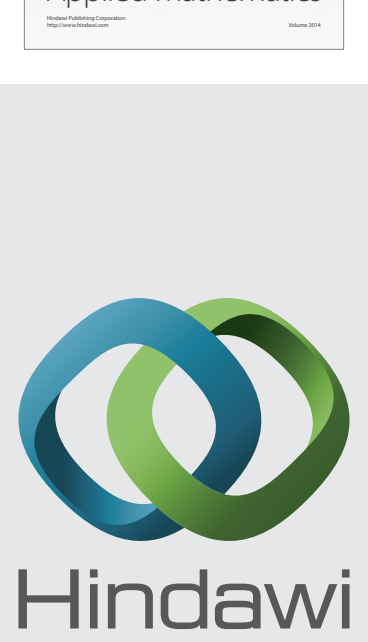

Submit your manuscripts at http://www.hindawi.com
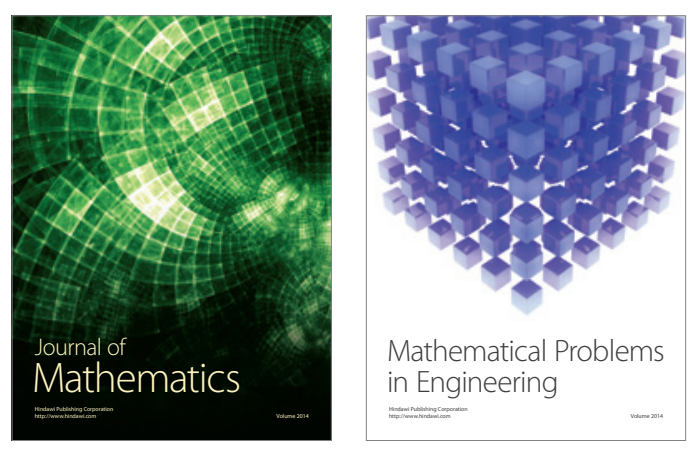

Mathematical Problems in Engineering
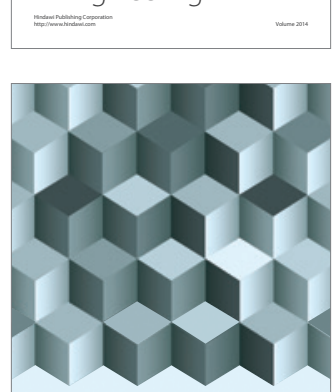

Journal of

Function Spaces
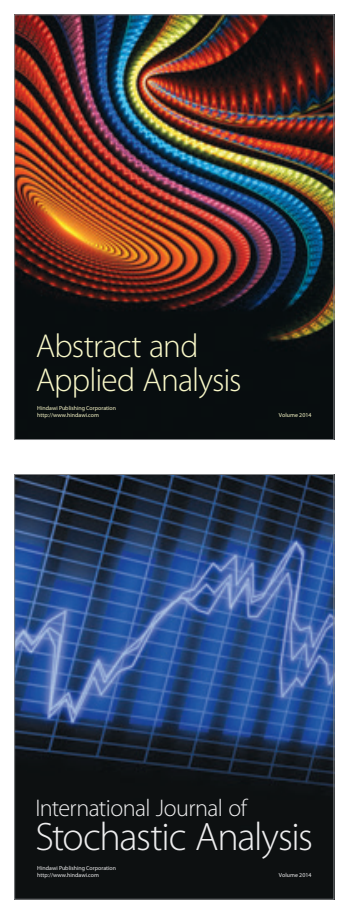

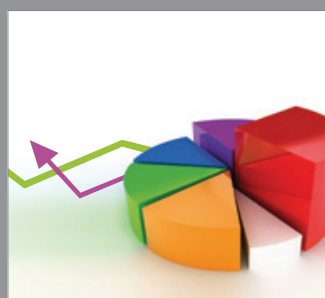

ournal of

Probability and Statistics

Promensencen
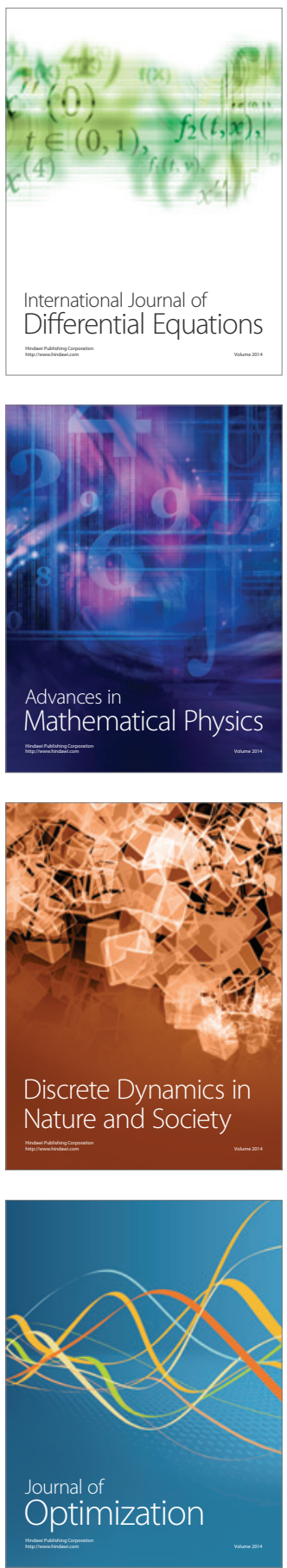